\title{
Article \\ Single cell center of mass for the analysis of BMP receptor heterodimers distributions
}

\author{
Hendrik Boog ${ }^{1,2} \oplus$, Rebecca Medda ${ }^{1,+}$ and E. Ada Cavalcanti-Adam ${ }^{1} \oplus *$ \\ 1 Department of Cellular Biophysics - Growth factor mechanobiology, Max-Planck-Institute for Medical \\ Research, Heidelberg, Germany; \\ 2 Institute for Pharmacy and Molecular Biotechnology (IPMB), Ruprecht-Karls-Universitaet Heidelberg, Im \\ Neuenheimer Feld 364, 69120 Heidelberg, Germany; \\ * Correspondence: Elisabetta.Cavalcanti-Adam@mpimf-heidelberg.mpg.de \\ † Current address: Department of Optical Nanoscopy, Max-Planck-Institute for Medical Research, Heidelberg, \\ Germany
}

\begin{abstract}
At the plasma membrane, transmembrane receptors are at the interface between cells and their environment. They allow sensing and transduction of chemical and mechanical extracellular signals. The spatial distribution of receptors and the specific recruitment of receptor subunits to the cell membrane is crucial for the regulation of signaling and cell behavior. However, it is challenging to define what regulates such spatial patterns for receptor localization, as cell shapes are extremely diverse when cells are maintained in standard culture conditions. Bone morphogenic protein receptors (BMPRs) are serine-threonine kinases, which build heteromeric complexes of BMPRI and II. These are especially interesting targets for receptor distribution studies, since the signaling pathways triggered by BMPR-complexes depends on their dimerization mode. They might exist as pre-formed complexes, or assemble upon binding of BMP, triggering cell signaling which leads to differentiation or migration. In this work we analyzed BMPR receptor distributions in single cells grown on micropatterns, which allows not only to control cell shape, but also the distribution of intracellular organelles and protein assemblies. We developed a script called ComRed (Center Of Mass Receptor Distribution), which uses center of mass calculations to analyze the shift and spread of receptor distributions according to the different cell shapes. ComRed was tested by simulating changes in experimental data, showing that shift and spread of distributions can be reliably detected. Our ComRed-based analysis of BMPR-complexes indicates that receptor distribution depends on cell polarization. The absence of a coordinated internalization after addition of BMP suggests that a rapid and continual recycling of BMPRs occurs. Receptor complexes formation and localization in cells induced by BMP might yield insights into the local regulation of different signaling pathways.
\end{abstract}

Keywords: receptor; distribution; BMP; BMPR; center of mass, micropattern

\section{Introduction}

Bone morphogenetic protein (BMP) is a growth factor involved in a plethora of cellular processes, like cell differentiation [1], adhesion and migration [2,3]. Bone morphogenetic protein receptors (BMPRs) belong to the serine-threonine kinase family and become activated upon binding of BMP molecules to their extracellular domains, triggering downstream signaling [4]. BMPRs are present in cells either as a preformed complex (PFC) of BMPRI and BMPRII, or a BMP-induced signaling complex (BISC). The PFC is already assembled in absence of the growth factor, while in the BISC, BMP binding to BMPRI triggers the assembly of the complex [4]. The two complexes activate two different signaling cascades, a Smad-dependent pathway triggered by the PFC and a non-Smad pathway triggered by 
the BISC [4,5]. In both pathways, the complexes internalize with or without the cargo [6,7]. The PFC is internalized by clathrin-mediated endocytosis [4,8], while the BISC is uptaken via caveolae structures [5,9]. Although BMPRs have been first identified in bone cells and cells of mesenchymal origin [10], these receptors are expressed in a variety of cells, including epithelial and endothelial cells [11,12]. In recent studies, the importance of BMPR-mediated signaling in endothelial cells has been highlighted for the maintenance of tissue homeostasis [13-15]. Among the commonly used endothelial cell lines, human umbilical cord vein endothelial cells (HUVECs) express both BMPRI and II but have a low expression of the BMPR Ia subtype (also called Alk3), in favor of BMPRIb $[12,16]$. These cells respond to BMP-2 treatment by increased migratory behavior and angiogenesis, suggesting that not only the specific expression of receptor subunits, but also the presence of the ligand and receptor spatial organization might regulate adhesion-mediated responses triggered by BMPs [12,16]. Analysis of receptor localization can be categorized into three different approaches - the analysis of receptor interactions, velocities, and spatial distribution. The analysis of receptor interactions, looks at short range co-occurrences or binding events of receptors. Established methods are for example fluorescence resonance energy transfer (FRET) [17,18], colocalization [19-21], or fluorescence (cross-)correlation spectroscopy [22]. An example for velocity studies is fluorescence recovery after photobleaching (FRAP) [23].Nohe, et al. used image correlation spectroscopy to analyze the receptor distributions of BMPRIa and BMPRII in COS7 and A431 cells. Here, a metric called cluster density, which is derived from image correlation spectroscopy, was used. BMPRIa formed clusters when cells were treated with BMP-2 for 2.5 hours and when in absence of BMPRII. Moreover, a kinase inactive variant of BMPRII also led to aggregation of BMPRIa [24]. Another study also used image correlation spectroscopy as well as FRET to show that BMPRI is shuffling towards BMPRII, which is located in precursors of clathrin-coated pits. Upon BMP induction, a significantly different distribution after inhibition of clathrin-mediated endocytosis could be observed [8]. However, the presented studies did not analyze spatial distributions within whole cells. A common problem of analyzing spatial distributions for whole cells are the non-uniform shapes and sizes of cells in standard culture conditions. Micropatterning techniques can restrict single cells to a uniform shape and size [25], which allows comparative analyses.

In the present study we developed the Center Of Mass REceptor Distribution (ComRed) script. ComRed analyzes the whole cell distributions of cellular receptors in fluorescence microscopy images, acquired after cell immunostaining. It uses data from upstream programs, for example Ilastik [26,27], that segment images automatically, to compute the center of mass of receptor clusters. As a frame of reference, the center of mass of the nucleus was taken. The functionality of ComRed was tested with data simulations. As receptor distributions of BMPRs were studied before [8,21,24], we also looked at BMPRs here using HUVECs, being an established primary cell model for the study of BMPRs $[12-16,21]$. As they increase their motility and invasion, as part of angiogenesis, upon BMP treatment $[12,15,16]$, spatial organization of BMPRs might play a substantial role in HUVECs. Additionally, HUVECs express BMPR proteins BMPRIb and BMPRII, while BMPRIa and Alk2 are only expressed slightly $[12,16]$. This simplifies the analysis of spatial distribution in HUVECs, as we expect no significant competition between BMPRIa and BMPRIb for BMPRII.

We compared receptor distributions in single cells that were grown on micropatterned substrates to control cell shape. We chose circle and crossbow shapes since cells on circular patterns adapt an unpolarized geometry due to isometric spreading, while cells on crossbow patterns adopt a polarized, migratory-like geometry $[25,28]$. Our results show that receptor distribution depends on cell polarization. Furthermore, we observed differences in BMPRIb and BMPRII distributions in unpolarized cells, indicating a preference for the BISC and the corresponding non-Smad pathways. ComRed is however not restricted to the analysis of BMPRs and in combination with micropatterning techniques it might be a useful tool to analyze the spatial distributions of any receptor of interest. 


\section{Results}

In this study the spatial distribution of BMPR subunits $\mathrm{Ib}$ and II was studied with ComRed, a script analyzing center of mass differences. The total intensity of objects corresponds to the weight in the center of mass calculations. Analogous to the mass, which is the product of density and volume, the total intensity is a product of the number of pixels or voxels (analogous to the volume) and the mean intensity (analogous to the density). Here we assumed, that the intensity of clusters is proportional to the number of receptors in the corresponding cluster. ComRed has two different metrics, based on center of mass calculations: 1) The center of mass of a given distribution, compared to a reference point, which might be inside or outside the cell. This is called reference metric. In this study the center of mass of the nucleus was chosen as reference point. 2) The mean distance of every object of a given distribution to the center of mass of the whole distribution, defined as spread metric. The comparison of the center of mass to a reference point corresponds to a shift in 3D to the reference, while the mean distance corresponds to the spread of a receptor distribution.

\subsection{ComRed-based analysis detects changes in experimental data}

Data simulations were used to show the strengths and limitations of ComRed. Simulated data was generated from experimental data by inverse transform sampling, i.e. a sampling method to generate a pool of random data points following an arbitrary distribution. Before the inverse transform sampling, the experimental data was altered by either shifting or spreading the function with a specific value $n$. A value of $n=0$ corresponds to no change, while higher $n$ values correspond to a larger shift or spread in comparison to the original distribution (see Data simulation). After the inverse transform sampling, the differences between the raw experimental data distribution and the sampled distribution were measured with the cosine and Hellinger distances, as well as a percentage histogram overlap. All required parameters ( $\mathrm{x}-, \mathrm{y}-$, and $\mathrm{z}$-coordinates, volume, and mean intensity) were simulated from experimental data of BMPRIb distributions from 72 confocal images of HUVEC cells grown on circular patterns having a diameter of $50 \mu \mathrm{m}$ to accomodate a single cell and control cell shape. First an inverse transform sampling mapping on the raw experimental data $(n=0)$ was done. Examples of simulated distributions in comparison with experimental data are shown in Figure 2. Similarity metrics for the simulation in comparison with experimental data (for $n=0$ ) are available in Figure A1. Subsequently the simulated data with n-values ranging from 0 to 2 were analyzed and plotted with ComRed. Figure 5 and Figure 6 show a summary of the data simulation results, when compared to experimental data. In addition, experimental datasets were randomly split into two groups, which were compared to each other. The comparison of randomly split groups did not yield any significant differences in any of the test cases (see Figure A2 and Figure A3). The reference metric shows significant differences for shifted data with $n \geq 0.2$, but no significant differences for data spread. This shows that the reference metric is detecting changes only on the distance of distributions to their reference point. The spread metric shows significant differences for data distribution with $n \geq 0.2$ For the shifted data it shows significant differences (when applying a threshold of $\mathrm{p}<0.05$ ) for $n=0, n=0.2$, and $n=1$. So, while the spread metric behaves as expected, for spreading distributions, it does not for the shifting of distributions. However, the distribution of mean distances of objects to their center of mass is much smaller for simulated data than for experimental data (see Figure 6). These differences are not observed, when comparing data split into two random groups (see Figure A3). Therefore, the significant differences in shifted data might be an artifact due to the simulation behavior. ComReds reference metric is robustly detecting changes in the shift of a distribution, but not its spread. The spread metric on the other hand is robustly detecting differences in spread, but might also be sensitive to spread changes of the shift metric. 
a
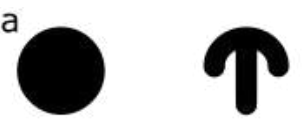

T
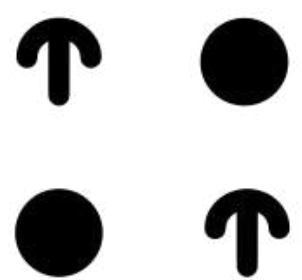

C

Calculation of receptors COM

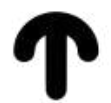

Calculation of nucleus COM

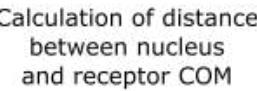

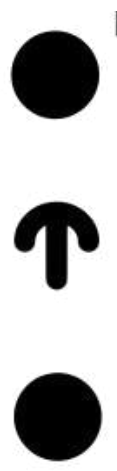

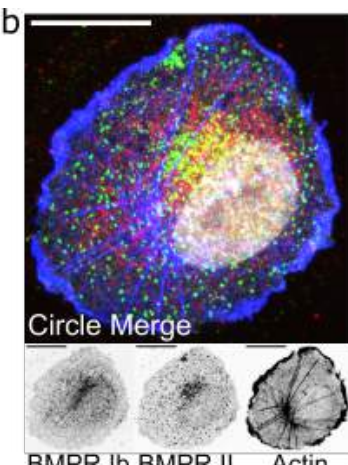

BMPR

Reference metric

d Spread metric
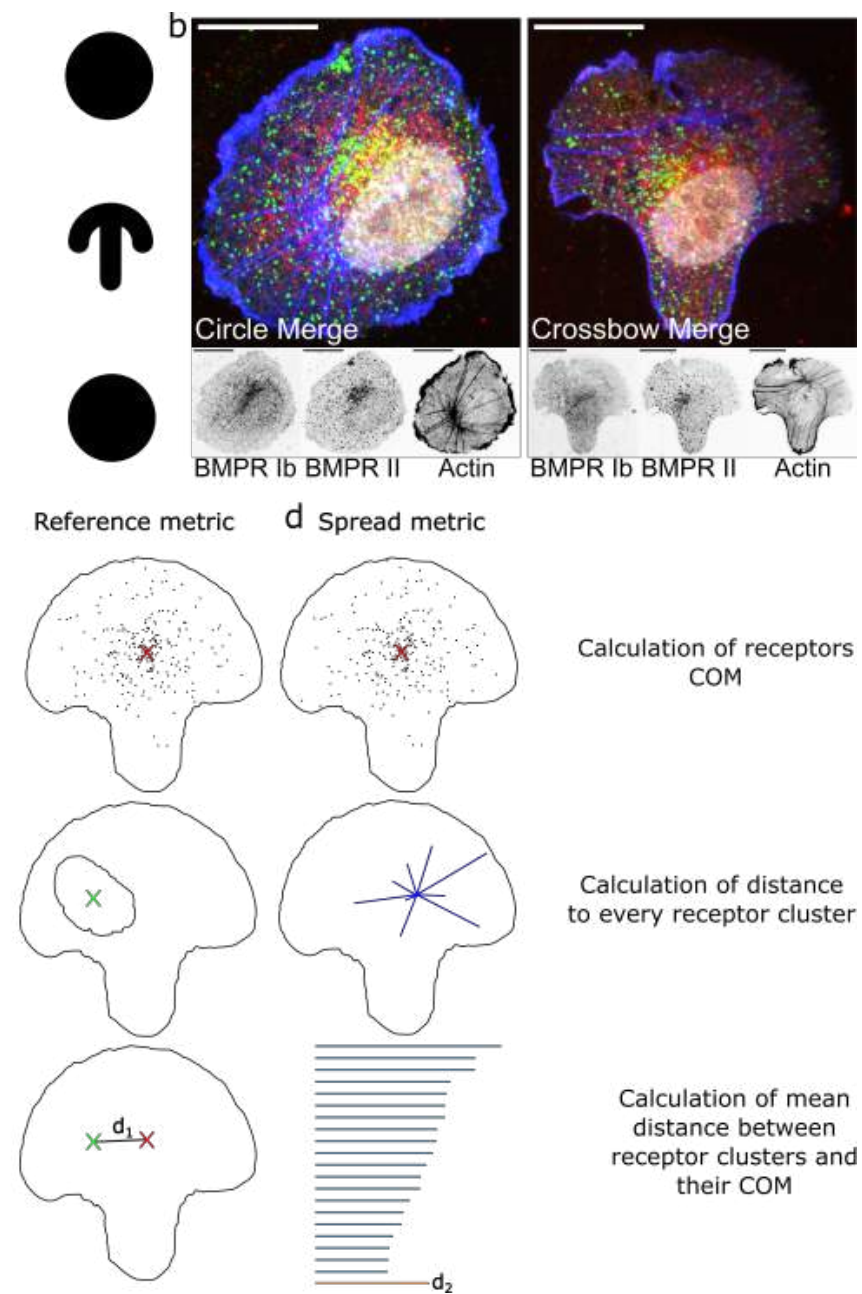

Calculation of receptors COM

Calculation of mean distance between
receptor clusters and

Calculation of distance to every receptor cluster

$$
\begin{gathered}
\text { receptor clusters } \\
\text { their COM }
\end{gathered}
$$

Figure 1. Outline of the experiments and the mode of operation of ComRed. a: Scheme of the circle and crossbow pattern. The white background is passivated with PLL-PEG, while the black patterns are micropatterned using a photomask in an UV-ozone cleaner and coated with fibronectin (see Photo-micropatterning). Circle diameter is $50 \mu \mathrm{m}$. b: Example confocal microscopy images of HUVECs seeded on a circle (left) and crossbow micropattern (right). Merge picture colors: Green: BMPRIb, Red: BMPRII, Blue: actin, White: nucleus. Scale bars in all confocal images are $20 \mu \mathrm{m}$ in length. c and d: Steps for the calculation of reference and spread metrics. For both metrics, first the center of mass of a receptor distribution is calculated. c: For the reference metric, a reference point is taken, in this case the center of mass of the nucleus. Afterwards the distance between the two center of mass points is calculated. d: For the spread metric, after determination of the center of mass of the receptor distribution, the distance to every found accumulation is calculated and the mean of these distances is taken. 


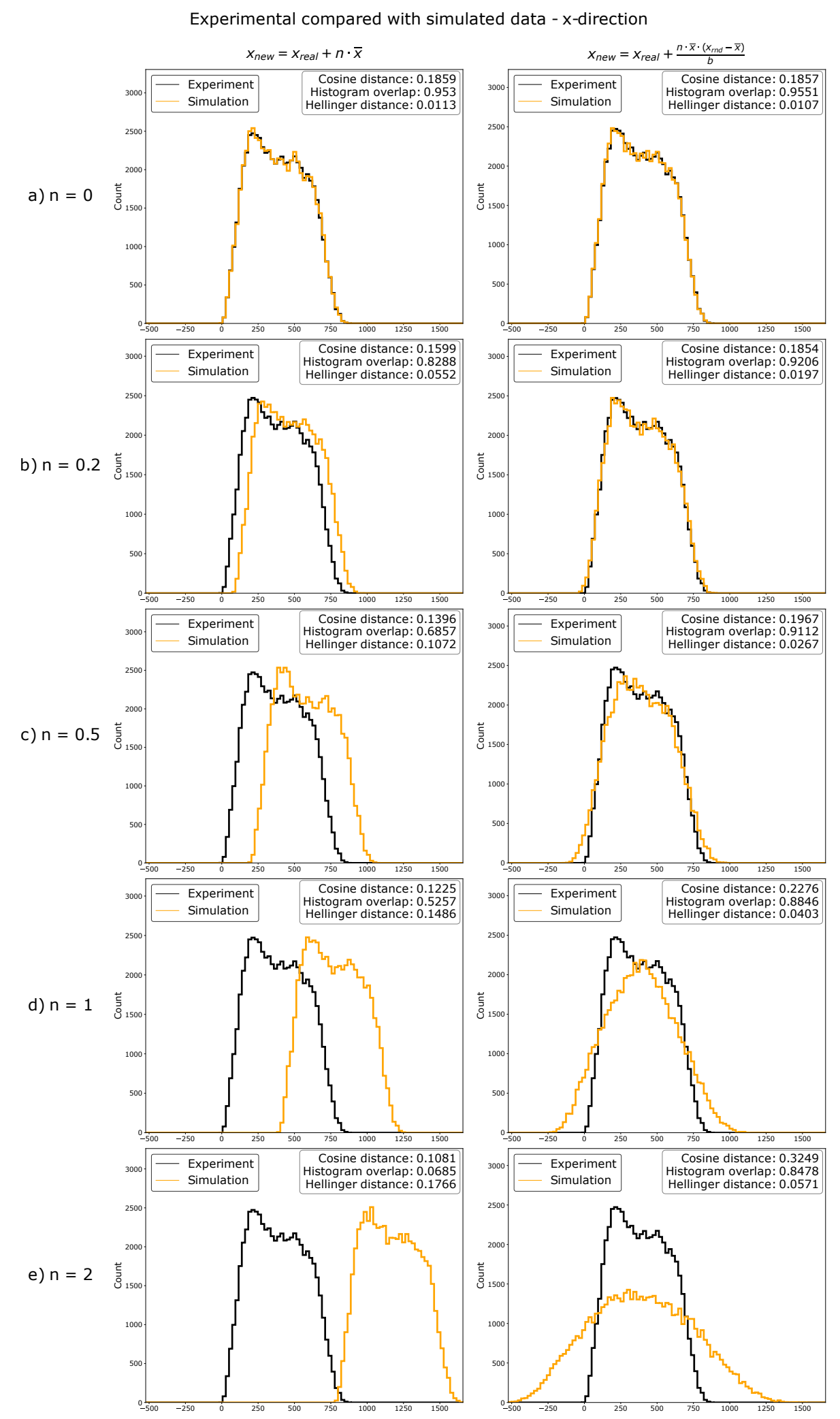

Figure 2. Overlaid boxplots of experimental data (black) and simulated data (orange) transformed with the factor $n$. Experimental data was used to generate simulated data, as explained in Data simulation. Different magnitudes of changes (as indicated with their $n$-value) were tested. The cosine distance and Hellinger distance, as well as the histogram overlap were calculated. This shows that the data can be effectively simulated by inverse transform sampling. Distributions can be effectively shifted or spreaded with using the functions $x_{\text {new }}=x_{\exp }+n \cdot \bar{x}$ (for shifting of distributions) or $x_{\text {new }}=x_{\text {exp }}+\frac{n \cdot \bar{x} \cdot\left(x_{r n d}-\bar{x}\right)}{b}$ (for spreading of distributions) before inverse transform sampling. 

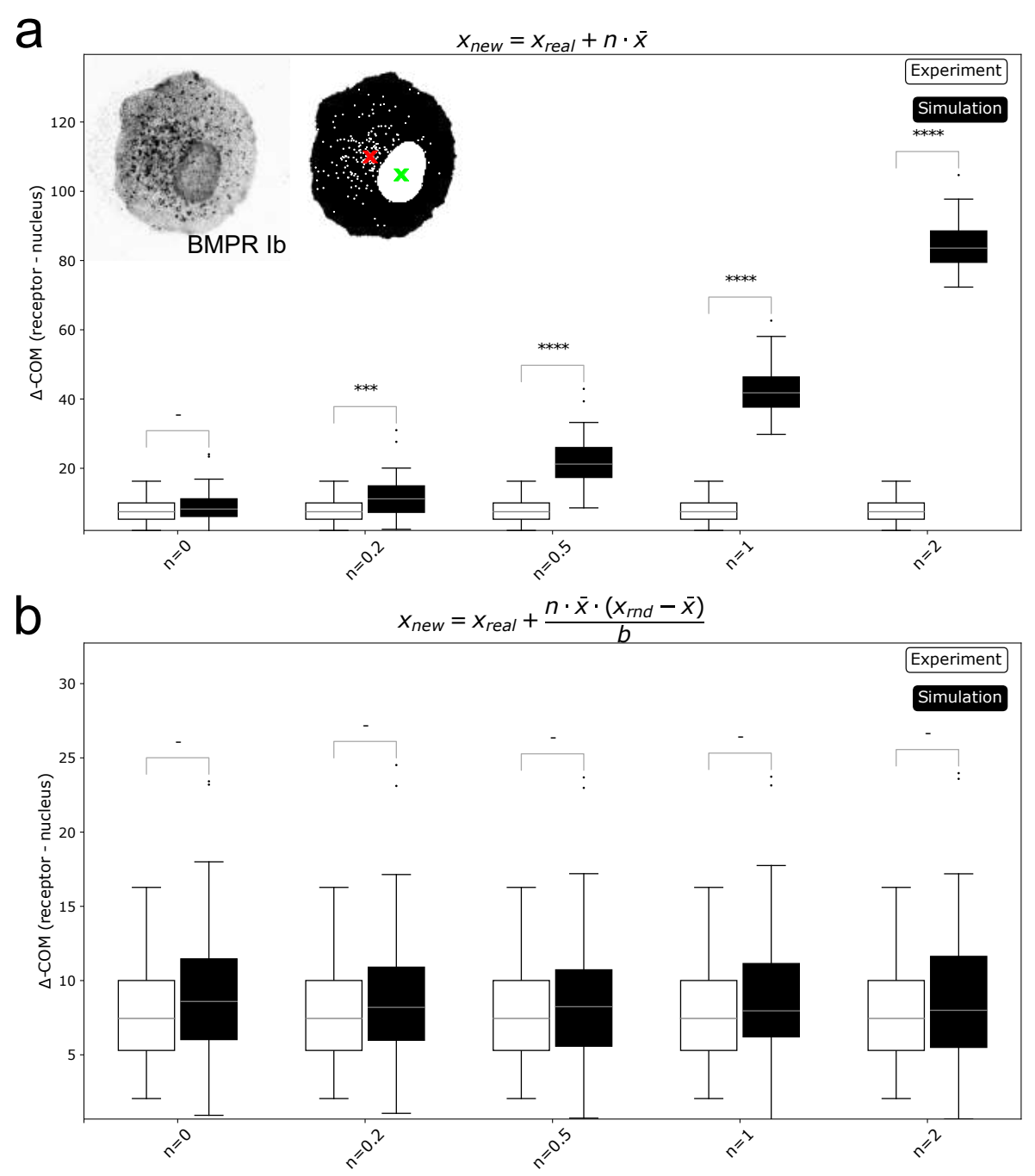

Figure 3. Comparison of simulatated and experimental data for the reference metric. a: Simulations of a shift in distributions $\left(x_{n e w}=x_{\text {real }}+n \cdot \bar{x}\right)$. All tested values for $\mathrm{n}>0$ showed significant differences. $\mathrm{b}$ : Simulations of a spread in distributions $\left(x_{\text {new }}=x_{\text {real }}+\frac{n \cdot \bar{x} \cdot\left(x_{r n d}-\bar{x}\right)}{b}\right)$. Here, for all values of $\mathrm{n}$ no significant differences were found. This shows that the reference metric is senstive shifts in the COM position, but not to differences in the spread of distributions. 

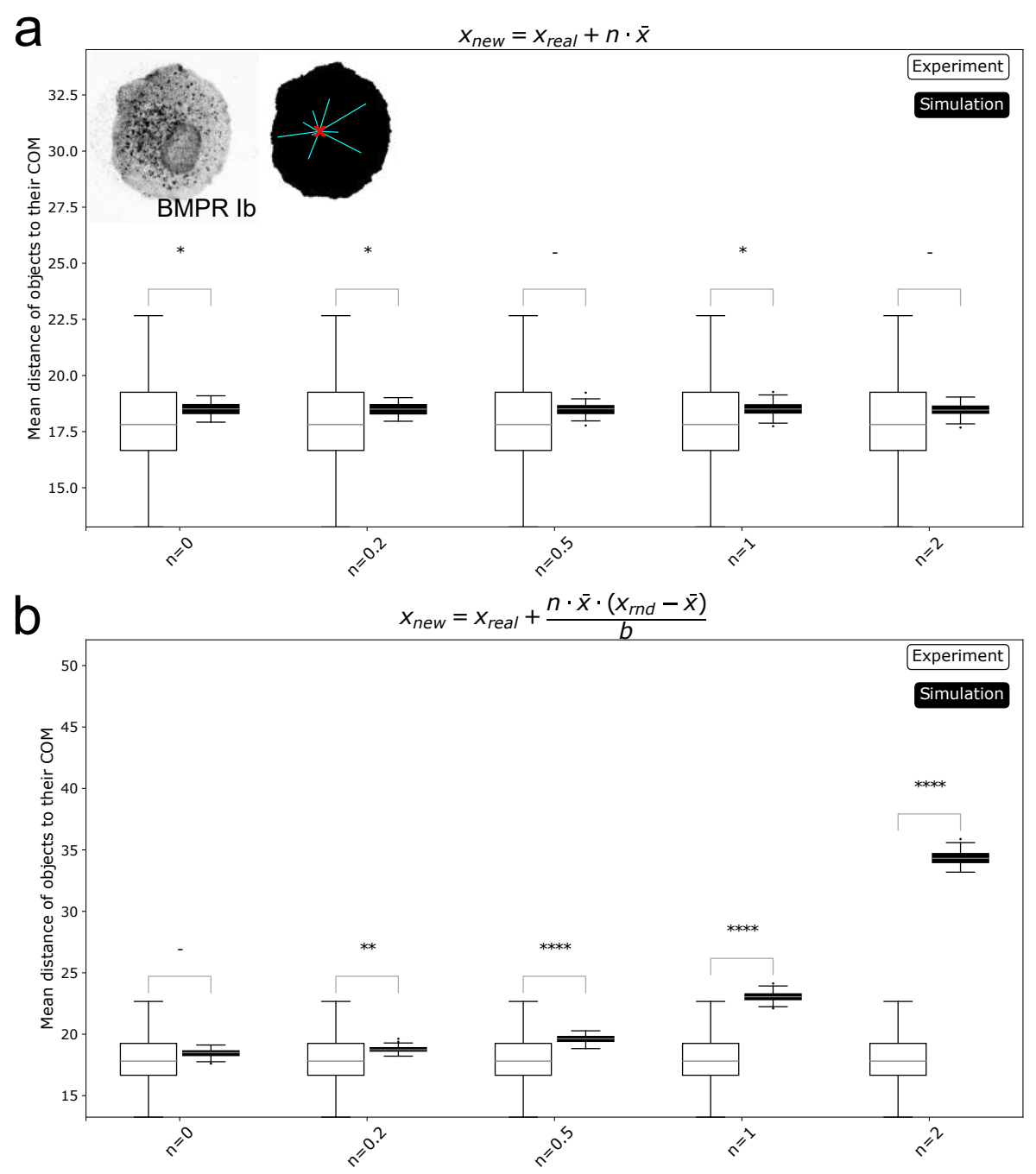

Figure 4. Comparison of simulatated and experimental data for the spread metric. a: Simulations of a shift in distributions $\left(x_{n e w}=x_{\text {real }}+n \cdot \bar{x}\right)$. b: Simulations of a spread in distributions $\left(x_{n e w}=\right.$ $\left.x_{\text {real }}+\frac{n \cdot \bar{x} \cdot\left(x_{r n d}-\bar{x}\right)}{b}\right)$. Here, for all values of $\mathrm{n}>0$ significant differences were found. This shows that the spread metric is sensitive for the spreading of distributions. 


\subsection{Cell geometry strongly influences BMPR subunit distribution}

After showing that the metrics used by ComRed are able to detect changes in localization and spread of receptors, ComRed was used to analyze distributions of BMPRIb and BMPRII in human umbilical vein endothelial cells (HUVECs). HUVECs were seeded on a micropatterned surface, which allows spreading of single cells into either a circular or a crossbow shape (see Figure 1). After cell spreading, the micropatterned adhesive regions were completely occupied. Cells were then exposed to BMP-2 added to the cell culture media for 15 minutes to trigger activation and internalization of the BMP-BMPRs complexes. Cells were fixed, immunostained, and imaged by confocal microscopy. Afterwards the images were analyzed with Ilastik and ComRed (for more details see Image processing and analysis and Center of mass calculations). Three pairs of comparisons were done. BMP-treated cells were compared with control cells (no addition of BMP), so differences in distribution due to relocalization of receptors induced by the presence of the ligand could be analyzed. Circle and crossbow shaped cells were compared, analyzing the differences in receptor distributions due to different cell geometries. BMPRIb and BMPRII distributions were compared, in order to infer, whether the two subunits might associate in a preformed complex (PFC) or in a BMP induced signaling complex (BISC), which both trigger different pathways. Should the distributions differ in shift or spread, the assembly of BISC might be preferred, as receptors are more likely to be in a dissociated state initially. A summary of the data produced by ComRed can be seen in Figure 5 and Figure 6 . The reference metric shows no significant shift of distributions, except for BMPRIb and BMPRII in control crossbow cells $(\mathrm{p}=0.025667)$. The spread metric shows three significant comparisons - In control circle cells BMPRIb is more spread than BMPRII, and in both control and BMP-treated populations, circle and crossbow cells have a different distribution of BMPRIb. BMPRIb is more spread out than BMPRII in control cells $\left(p=1.067 \cdot 10^{-3}\right)$. However, this difference was no longer present upon addition of $\mathrm{BMP}$, as in in the BMP induced case no significant difference between the receptor localizations could be found. This indicates that in HUVEC cells the BISC (and the corresponding Smad-independent pathway) might be preferred when cells have an unpolarized geometry, but not when in a polarized geometry, like the crossbow shape. Thus, the probability of BMP binding events, that lead to the activation of the Smad independent pathway, might be higher in control circle cells than in control crossbow cells. In BMP-treated cells, this discrepancy is no longer evident, as BMPRIb and BMPRII need to associate to trigger both signaling pathways. In control and BMP-induced cells, BMPRIb is more spread out in circle cells than in crossbow cells $\left(p=9.908 \cdot 10^{-7}\right.$ and $p=5.209 \cdot 10^{-5}$ for control and BMP comparisons respectively). Both receptors, BMPRIb and BMPRII are subjected to the same geometric constraints of the crossbow shape. If these changes were to depend only on the differences in cell geometries, also BMPRII would be more spread out in circular than in crossbow shaped cells. Therefore HUVEC cells might modulate the spread of BMPRIb based on polarization. Interestingly, there was no difference between the receptor distributions, when comparing control and BMP-treated cells. This suggests that a simultaneous internalization of the majority of receptors is unlikely.

Data generated with ComRed might be supplemented with external data. Here we wanted to complete our data with knowledge whether the induced complex or the preformed complex is the dominant mode of interaction with BMP, through image colocalization analysis. Colocalization was evaluated with JaCoP [20], an ImageJ extension. Both signaling complexes should behave differently in a colocalization analysis. In the PFC the receptors are in close vicinity, because they bind to each other before BMP is binding to the complex. If a cell only uses the preformed complex, there should be no difference in the colocalization between the induced and non-induced state, because the complexes are already formed. In the BMP-induced signaling complex both receptors are spatially separated, they only bind to each other when BMP is binding to BMPRI first. Both receptors might however be in close proximity, without binding - so that BISCs might appear like PFCs in the colocalization data. If however there is a significant difference between induced and non-induced states, there should be a preference for BISCs, as the receptor builds complexes only after BMP binding. Here two 
different scores were used for validation, Pearson's score (correlation) and Li's image colocalization quotient (Li's ICQ, colocalization). Four different comparison were done. Circle and crossbow cells were compared with and without BMP-treatment. In addition, scores for cells in presence or absence of BMP were compared for circle and crossbow shapes. The resulting data is presented in Figure 7. For the Pearson's score no significant difference could be detected in any of the comparisons. For Li's ICQ, two significant differences were detected. One between circle and crossbow cells in BMP- treated cells $(p=0.0079897)$ and one between control and BMP-treated circle cells $(p=0.0221327)$. The difference between control and BMP states agrees with the earlier results, where circle cells have a more spread out BMPRIb, where the difference is vanishing after 15 minutes of BMP induction. The results from Li's ICQ are however not reproducible with Pearson's Score.

\section{Discussion}

In this study we observed differences in distributions between BMPRIb and BMPRII in circular, but not in crossbow cells. Additionally this difference was no longer present after induction with BMP2, indicating the formation of fully functional signaling complexes from BISCs. Benn et al. showed that the Smad-dependent response of HUVEC cells to BMP2 is very low, while there is a significant response in the non-Smad dependent pathway. Other cell types (human aortic endothelial cells and human pulmonary microvascular endothelial cells) react with both Smad-dependent and non-Smad dependent responses. It seems that the PFC for BMP-2 is strongly mediated by BMPRIa, which is expressed only weakly in HUVECs [12]. There is also a difference between circular and crossbow cells BMPRIb distribution. This might indicate that the preference for a specific pathway might not only be dependent on the type of cell and receptor, but also on the polarization of the cell. Although fully assembled BMPR complexes are known to internalize [29], their distributions are not compacting towards their center of mass. Therefore we did not observe any coordinated internalization behavior in our experiments. It is unlikely, that internalization requires a longer timeframe, than the 15 minutes of incubation we did in our study. In a study analyzing the internalization behavior of BMP (and as such the correspoding BMPRs), a peak could be reached already after 15 minutes [29]. Therefore we propose that BMPRs might be recycled constantly. As receptors are internalized, other receptors are removed from an intracellular depot and are assembled in the cellular membrane. This replenishes the internalizing receptors and the spread with or without BMP induction does not change. A similar behavior can be seen in other membrane-bound proteins and receptors [30], like transforming growth factor $\beta$ receptors (TGF $\beta$ R) [31]. Mitchell et al. have shown that TGF $\beta$ Rs are constantly internalized and relocalized to the surface, even in the absence of ligand [31]. TGF $\beta$ receptors and BMPRs are structurally related. As such they form similar complexes of type I and type II receptors [32,33], as well as the internalization via clathrin-coated pit or calveolae [34]. Studies in C. elegans have shown that deletion of key proteins in the recycling pathway lead to an accumulation of BMPR homologous proteins in vesicles with simultaneous depletion in the cell membrane [35]. These studies back up the claim of recycling BMPRs, but, to our knowledge, a definite analysis of the recycling behavior of BMPRs in mammalian cells has not been done yet.

ComRed can be a useful tool for analysis of receptor distributions and hypothesis generation. We could show that the program can produce robust data, by testing on data simulations and real data comparison and how to generate meaningful hypotheses from ComReds generated data. Like in BMPRs the spatial distribution of other cellular receptors might have a significant impact on underlying cellular processes. Therefore ComRed might be used as an early analysis for future studies. After this initial analysis other methods like FRET, FRAP, fluorescence cross-spectroscopy or others might be chosen to gain a deeper understanding of the receptors and the processes they are involved in. 


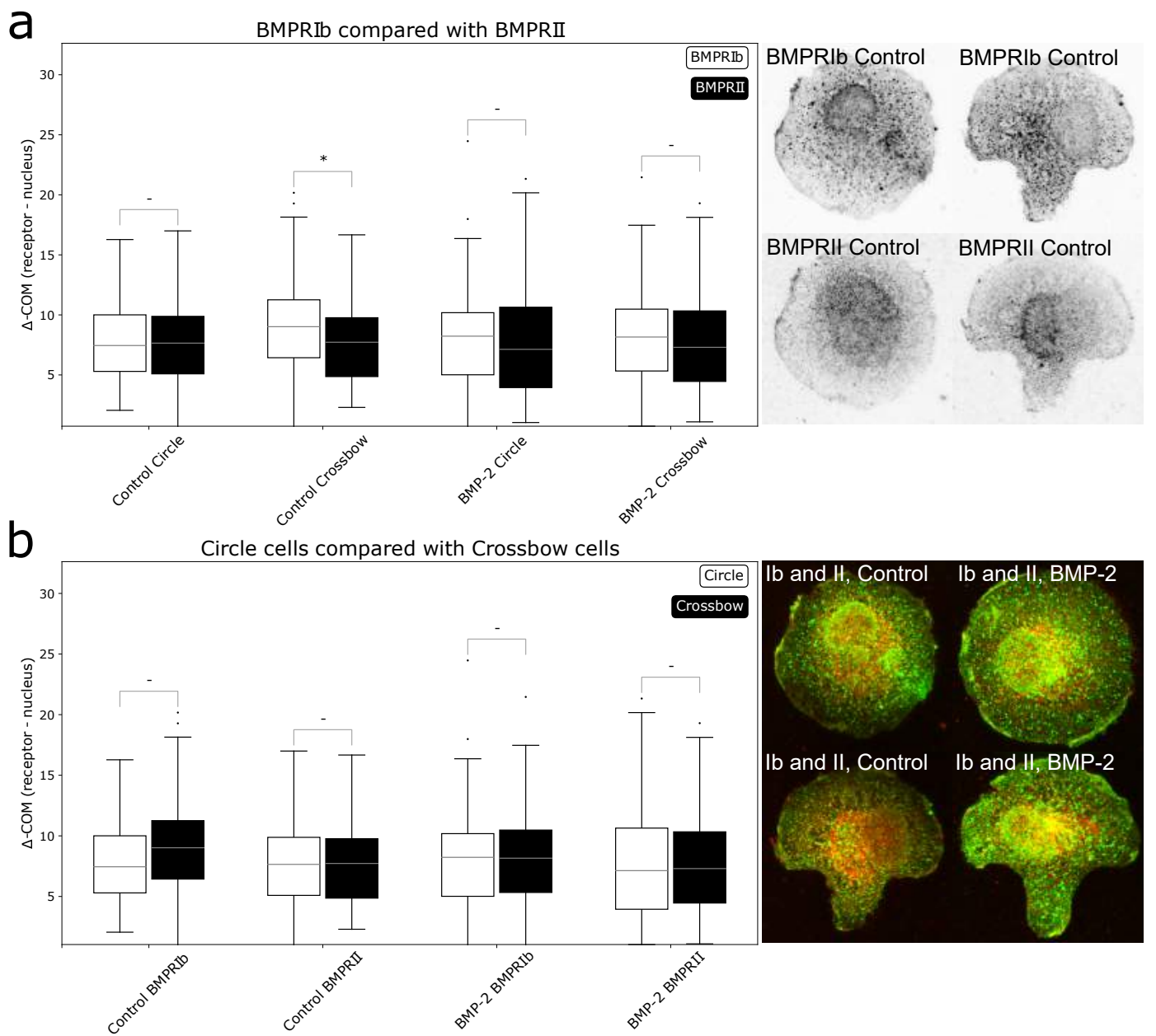

C Control cells compared with BMP-2 induced cells

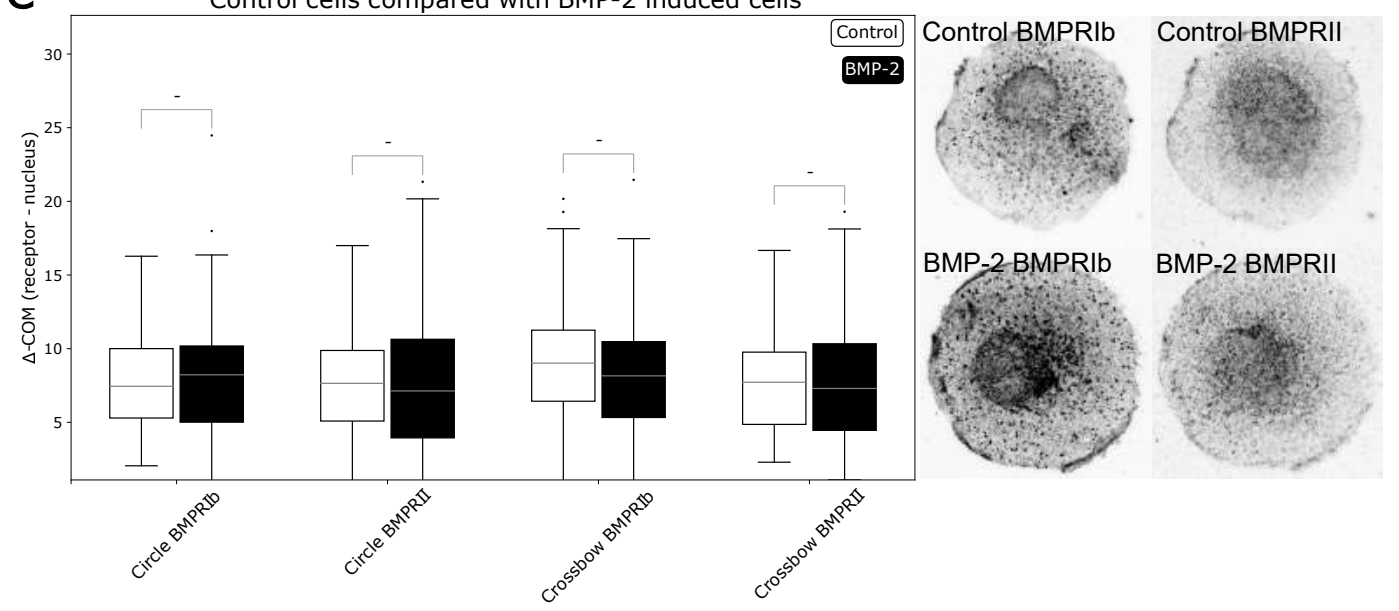

Figure 5. Comparison of receptor distributions of BMPR subunits in HUVECs. Reference metric data shown. a: Distributions of BMPRIb compared to distributions of BMPRII. For in control conditions differences could be found in crossbow cells $(p \approx 0.026)$. b: Circle compared with crossbow cells. No significant differences could be found. Color code for the confocal images: Green: BMPRIb, red: BMPRII. c: Control cells compared with cells that were induced with BMP-2. No significant differences were found. Except for BMPRIb and II in control crossbow cells, receptor distributions are not shifted against each other. 


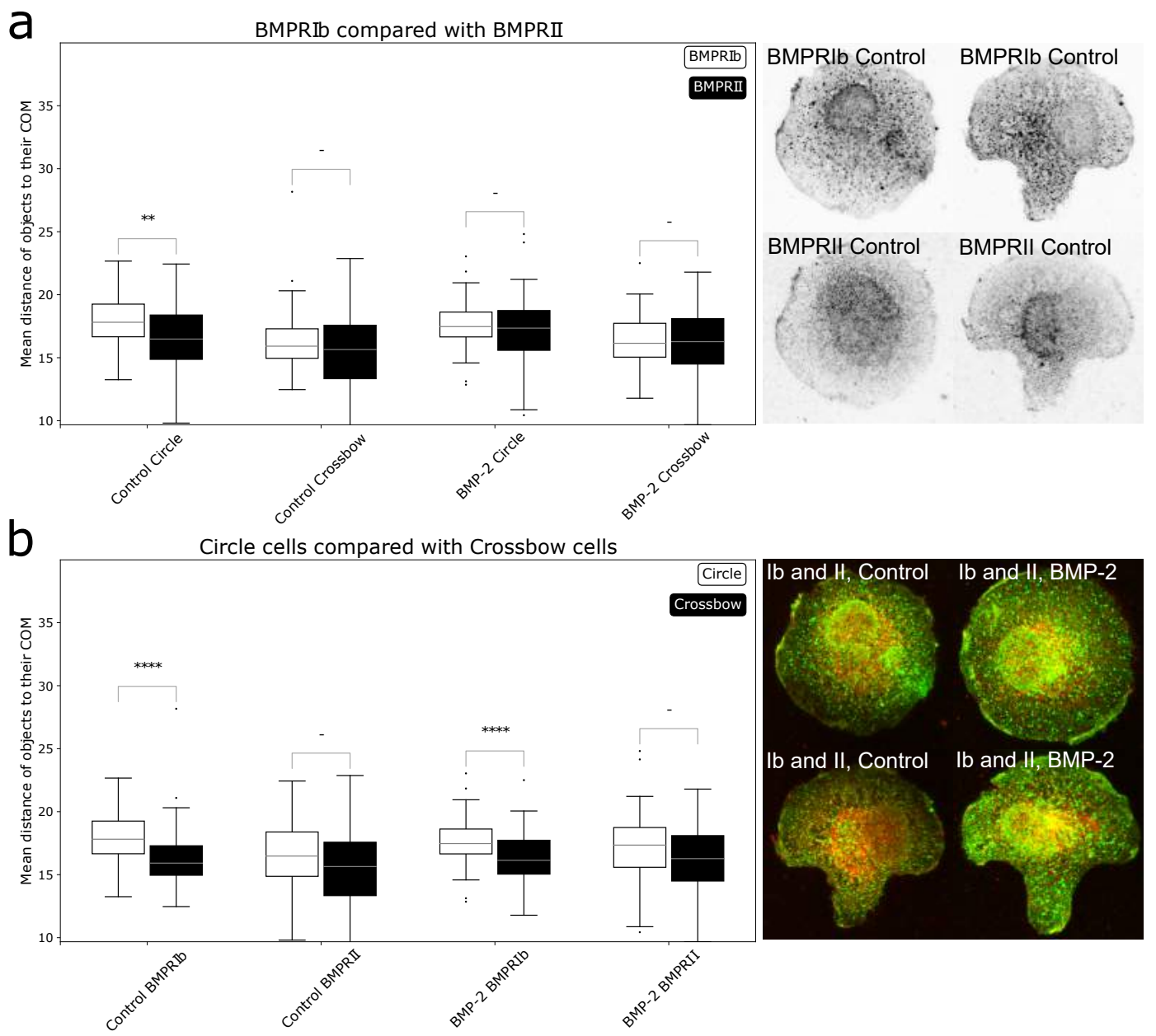

C Control cells compared with BMP-2 induced cells

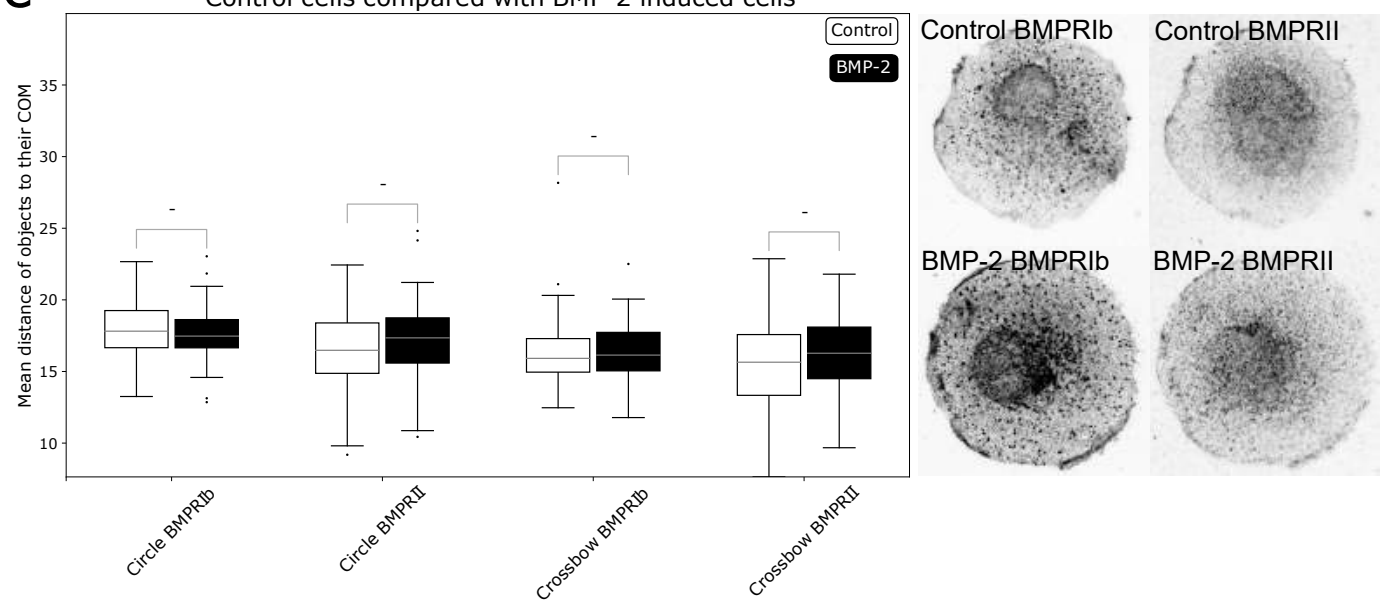

Figure 6. Comparison of receptor distributions of BMPR subunits in HUVECs. Spread metric data shown. a: Distributions of BMPRIb compared to distributions of BMPRII. b: Circle compared with crossbow cells. Color code for the confocal images: Green: BMPRIb, red: BMPRII. c: Control cells compared with cells that were induced with BMP-2. Control and BMP induced conditions do not show a significant difference, this might indicate, that the majority of BMPRs is not internalizing simulataneously after addition of BMP. Moreover BMPRIb is more spead out in circle than in crossbow cells, both under control and BMP conditions ( $p \approx 10^{-6}$ and $p \approx 5 \cdot 10^{-5}$ respectively). Also there was differences found between BMPRIb and BMPRII $\left(p \approx 10^{-3}\right)$ in circular cells without BMP, indicating a preference for the BISC. 

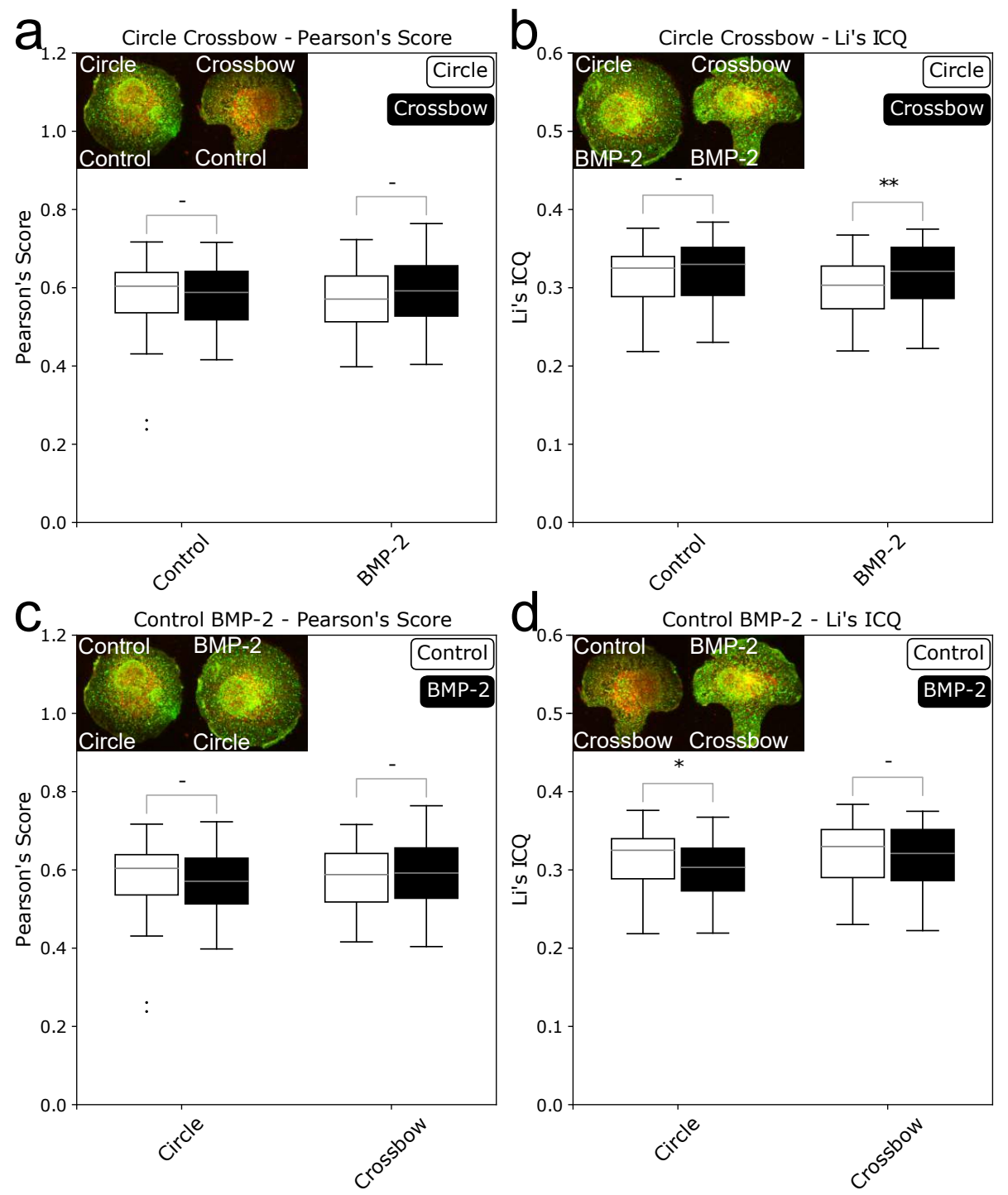

Figure 7. Comparison boxplots of colocalization values of BMPRIb and BMPRII in HUVECs. a and b: Colocalization values of circle and crossbow cells +/- BMP-2, using Pearson's score (a) and Li's ICQ (b). $\mathrm{c}$ and d: Colocalization values of control and BMP-2 induced cells in circle and crossbow cells, using Pearson's score (c) and Li's ICQ (d). Confocal images of HUVECs on circle- and crossbow patterns were analyzed with the JaCoP imageJ extension [20]. Changes in colocalization between induced an uninduced cells indicate that the BMP-induced signaling complex is preferably used. While no change in colocalization indicates, that the preformed complex is predominantly used. 


\section{Materials and Methods}

\subsection{Photo-micropatterning}

20x20 mm² or $24 \times 24 \mathrm{~mm}^{2}$ coverslips (Carl Roth, Germany) were sonication-bathed (Sonorex Super RK102H, Bandelin, Germany) in ethanol for 30 minutes and dried. The surfaces were passivated with $0.1 \mathrm{mg} / \mathrm{ml}$ poly-L-lysine-polyethylene-glycol (PLL-PEG, Surface solutions, Switzerland) in HEPES solution, before photo-micropatterning with a UV-ozone cleaner (Model 342-220, Jelight Company Inc., USA). The surfaces were subsequently equilibrated in PBS and incubated with $0.0625 \mu \mathrm{g} / \mathrm{cm}^{2}$ human cellular fibronectin (Sigma Aldrich, USA) in PBS for one hour, before washing with PBS. For the experiments a circle- and crossbow-pattern was used, where every spot has a diameter of $50 \mu \mathrm{m}$, to ensure only one cell spreads on every spot.

\subsection{Cell culture}

Human umbilical vein endothelial cells (HUVECs, PromoCell, Germany) were cultured in T25 flasks (Greiner Bio One, Germany) using Endothelial cell basal medium 1/2 (PromoCell, Germany). They were split using Accutase (Thermo Fisher Scientific, USA) for detachment. Cells were seeded on 20x20 mm $\mathrm{mm}^{2}$ or $24 \times 24 \mathrm{~mm}^{2}$ coverslips (Carl Roth, Germany) in 6-well plates (Thermo Fisher Scientific, USA). Cells were fixed using 4\% (w/V) paraformaldehyde (Sigma Aldrich, USA) in PBS at room temperature for 15 minutes.

\subsection{Immunofluorescence staining}

Paraformaldehyde fixed cells on coverslips were permeabilized with $0.1 \%$ (v/v) Triton-X-100 (Sigma Aldrich, USA) in PBS (V/V) solution for 10 minutes. Subsequently the surfaces were blocked with $2 \%$ BSA (Carl Roth, Germany) in PBS solution. The surfaces were incubated with primary, then secondary antibody solution for one hour. Actin was stained using Phalloidin-TRITC (P1951, Sigma Aldrich, USA) at $5 \mu \mathrm{g} / \mathrm{ml}$, BMPRIb was stained using anti-BMPRIb mouse mIgG (MAB5051, R\&D Systems, USA) at $5 \mu \mathrm{g} / \mathrm{ml}$, p-Smad was stained using anti p-Smad1/5 (Ser463/465) rabbit IgG (9516, Cell signaling, USA) at $1 \mu \mathrm{g} / \mathrm{ml}$, BMPRII was stained using anti-BMPRII rabbit pIgG (AP11864PU-N, Acris Antibodies, Germany) at $2.5 \mu \mathrm{g} / \mathrm{ml}$. As secondary antibodies we used Alexa647 goat anti-rabbit IgG (A-21244, Thermo Fisher Scientific, USA), and Alexa488 goat anti-mouse IgG (A-11029, Thermo Fisher Scientific, USA) at $10 \mu \mathrm{g} / \mathrm{ml}$. After primary and secondary antibody staining, the coverslips were washed in 2\% BSA in PBS. Stained coverslips were embedded in mowiol (Carl Roth, Germany) supplemented with DABCO (Sigma Aldrich, USA) and $1 \mu \mathrm{g} / \mathrm{ml}$ DAPI (Thermo Fisher Scientific, USA).

\subsection{Microscopy}

Confocal images were procured using a Zeiss LSM880 (Zeiss, Germany). The LSM880 was used for generating z-stack confocal images of single cells with a $63 x$ oil objective. Only cells with the desired geometry (circle or crossbow) were imaged. Micropattern spots with more than one attached cell were excluded.

4.5. Seeding human umbilical vein endothelial cells on micropatterns and induction with bone morphogenetic protein $2(B M P-2)$

Human umbilical vein endothelial cells (HUVECs) were cultivated according to the Cell culture section. HUVECs were seeded on circle crossbow micropatterned surfaces (see Photo-micropatterning). Cells were allowed to spread on the micropattern for 45 minutes. Afterwards either bone morphogenic protein 2 (BMP-2) derived from chinese hamster ovary cells (CHO cells; R \& D Systems, USA) to a final concentration of $20 \mathrm{nM}$ or vehicle was added (control condition) and the cells were incubated for another 15 minutes. Then, the cells were fixed with paraformaldehyde and subsequently immunostained (see Cell culture and Immunofluorescence staining). 


\subsection{Image processing and analysis}

Before use of the center of mass calculations confocal mircroscopy pictures were processed with two different programs: ImageJs Fiji distribution (Version 1.51) [36] and ilastik (Version 1.2) [26,27]. Fiji was used for image preprocessing, where pictures were converted to grayscale images and contrast was adjusted. ilastik, a machine learning framework for biomedical imaging, was used for object detection of receptor accumulations. The 3D positions, volumes and mean intensities for every receptor accumulation were determined. Colocalization was calculated with the JaCoP imageJ extension [20]. For the colocalization the Pearson's Score and Li's ICQ were used. For Li's Score first calculates $\left(A_{i}-a\right)\left(B_{i}-b\right)$, where $A_{i}$ and $B_{i}$ are pixel intensities of the same pixel in two channels and $a$ and $b$ are the means of intensites in one channel in the whole picture. Then the quotient of the positive values to all values is calculated. In the end also 0.5 is subtracted from the result, so the ICQ ranges between -0.5 and 0.5 [19]. Pearson's Score is a correlation score, which is defined as $P=\frac{\sum\left(A_{i}-a\right)\left(B_{i}-b\right)}{\sqrt{\sum\left(A_{i}-a\right)^{2}} \cdot \sqrt{\sum\left(B_{i}-b\right)^{2}}}$, ranges from 0 to 1 .

\subsection{Center of mass calculations}

The general formula for center of mass calculations is given as: $r=\frac{\sum m_{i} \cdot r_{i}}{\sum m_{i}}$. Where $m_{i}$ is the mass of every object and $r_{i}$ is its position in one spatial direction and $\mathrm{r}$ is the position of the center of mass. For pictures the real mass is substituted by the virtual mass given as: $v_{i}=\bar{I}_{i, g} \cdot n_{i}$, where $\bar{I}_{i, g}$ is the mean intensity of the object normalized over the mean intensity of the picture $\left(\bar{I}_{i, g}=\frac{I_{i}}{I_{g}}\right.$ and $n_{i}$ is the object volume in voxels, in our case $0.0037 \mu \mathrm{m}^{3}$ ). The virtual mass $v_{i}=\bar{I}_{i, g} \cdot n_{i}$ is analogous to the real world mass, which is given by density times volume. Inserted into the original center of mass formula $I_{g}$ cancels out, leaving $r=\frac{\sum I_{i} \cdot n_{i} \cdot r_{i}}{\sum I_{i} \cdot n_{i}}$. Pictures might differ in size (physical dimensions, and/or pixel volume, and/or pixel number), and cell orientation and position. Therefore object coordinates or center of mass coordinates need to be compared to a frame of reference. $d=r_{o}-r_{r}$ where $d$ is the difference between an object and its reference, $r_{r}$ is the reference position and $r_{o}$ is the object position. After the positions for all spatial directions were calculated the arithmetic mean might be calculated $\bar{d}=\sqrt{\left(d_{x}\right)^{2}+\left(d_{y}\right)^{2}+\left(d_{z}\right)^{2}}$. In the end the difference in pixels might can be converted into a length scale of choice (here micrometer). In this study we used two metrics - a reference metric and a spread metric. In the reference metric, the object (as indicated by $r_{o}$ is the center of mass of a receptor distribution, while the reference $\left(r_{r}\right)$ is the center of mass of the cells nucleus). In the spread metric, the object is every receptor accumulation detected (so every spot on the picture would be one object) and the reference is the center of mass of all found accumulations of the same receptor type. Then, the mean of the spread values was calculated for every cell. The current version of ComRed is available through https://github.com/hendrikboog/comred.

\subsection{Statistics}

Statistics were done with pythons scipy package using a two-sided Mann-Whitney-U test.

\subsection{Data simulation}

In order to test the boundaries of the data analysis tool, data was simulated by mimicking experimental data. This was done with inverse transform sampling. With this method one might generate random numbers from an arbitrary probability distribution. In this case the probability distribution is the distribution of the position of single receptor accumulations, their volumes, or mean intensities. To get a random distribution the equation $F\left(F^{-1}(y)\right)=y$ needs to be solved for $F^{-1}(y)$, where $F(x)$ is the non-inverse distribution and $F^{-1}(y)$ is the inverse transform of the distribution. The inverse transform of a probability distribution can now be fed with random variables from a uniform 
distribution between 0 and 1 to get random numbers that fit the distribution $F(x)$. As a subsequent step the input data might be altered, so that different cases of experimental versus model data can be examined. Here we focused on displacement of the function and a change in spread (effectively "flattening") of the function. These two cases represent a change in the center of mass and widely distributed receptors, respectively. For the first, every experimental input data point was altered with $x_{n e w}=x_{\text {exp }}+n \cdot \bar{x}$, where $x_{n e w}$ is the altered data point, $x_{\text {exp }}$ is the read in datapoint from experimental data, $n$ is an arbitrary real number and $\bar{x}$ is the mean of all experimental data points $x_{i}$. For the change in spread the following formula was used: $x_{n e w}=x_{\text {exp }}+\frac{n \cdot \bar{x} \cdot\left(x_{r n d}-\bar{x}\right)}{b}$, where $x_{r n d}$ is a random number drawn from an inverse transform sampling of the experimental data and $b$ is the longest distance between the mean of data and the outermost data point. Afterwards simulated data was produced by inverse transform sampling of the altered data. Both receptor distributions and reference points were simulated. Reference points were always simulated with $n=0$, while the receptors were simulated with n-values from 0 to 2 . Discrepancies between simulated and experimental data were analyzed with the cosine distance, a percentual histogram overlap and the Hellinger distance. The cosine distance is the cosine of the angle between two vectors of data. The distance ranges between 0 and 1 , where 0 is no change. The cosine distance is independent of the vectors length. The histogram overlap is the normalized integral between both histogram functions. A value of 1 is a perfect overlap and a value of 0 means no overlap. The Hellinger distance for discrete distributions is defined as $H(P, Q)=\frac{1}{\sqrt{2}} \sqrt{\sum_{i=1}^{k}\left(\sqrt{p_{i}}-\sqrt{q_{i}}\right)^{2}}$, the larger the Hellinger distance, the more distinct the two distributions are.

Author Contributions: Conceptualization, H.B., R.M. and A.C.; methodology, H.B.; software, H.B.; validation, H.B., R.M. and A.C.; formal analysis, H.B.; investigation, H.B. and R.M.; resources, A.C.; data curation, H.B.; writing-original draft preparation, H.B. and R.M.; writing-review and editing, H.B., R.M. and A.C.; visualization, H.B.; supervision, R.M. and A.C.; project administration, A.C.; funding acquisition, A.C. All authors have read and agreed to the published version of the manuscript.

Funding: This research was funded by the Max Planck Society and by the German Science Foundation (DFG TRR79 TPB05).

Acknowledgments: We thank Nico Kusterer for helpful discussions.

Conflicts of Interest: The authors declare no conflict of interest.

\section{Abbreviations}

The following abbreviations are used in this manuscript:

$\begin{array}{ll}\text { BISC } & \text { BMP induced signaling complex } \\ \text { BMP } & \text { Bone morphogenetic protein } \\ \text { BMPR } & \text { Bone morphogenetic protein receptor } \\ \text { ComRed } & \text { Center Of Mass REeptor Distribution } \\ \text { DAPI } & \text { 4',6-Diamidine-2'-phenylindole-dihydrochloride } \\ \text { HUVEC } & \text { Human umbilical vein endothelial cell } \\ \text { PFC } & \text { Preformed complex }\end{array}$

\section{Appendix A}

Appendix A.1

\section{References}

1. Kang, Q.; Song, W.X.; Luo, Q.; Tang, N.; Luo, J.; Luo, X.; Chen, J.; Bi, Y.; He, B.C.; Park, J.K.; Jiang, W.; Tang, Y.; Huang, J.; Su, Y.; Zhu, G.H.; He, Y.; Yin, H.; Hu, Z.; Wang, Y.; Chen, L.; Zuo, G.W.; Pan, X.; Shen, J.; Vokes, T.; Reid, R.R.; Haydon, R.C.; Luu, H.H.; He, T.C. A comprehensive analysis of the dual roles of 
Control Circle BMPRIb simulated versus experimental data Inverse Transform Sampling check shift $n=0.0$
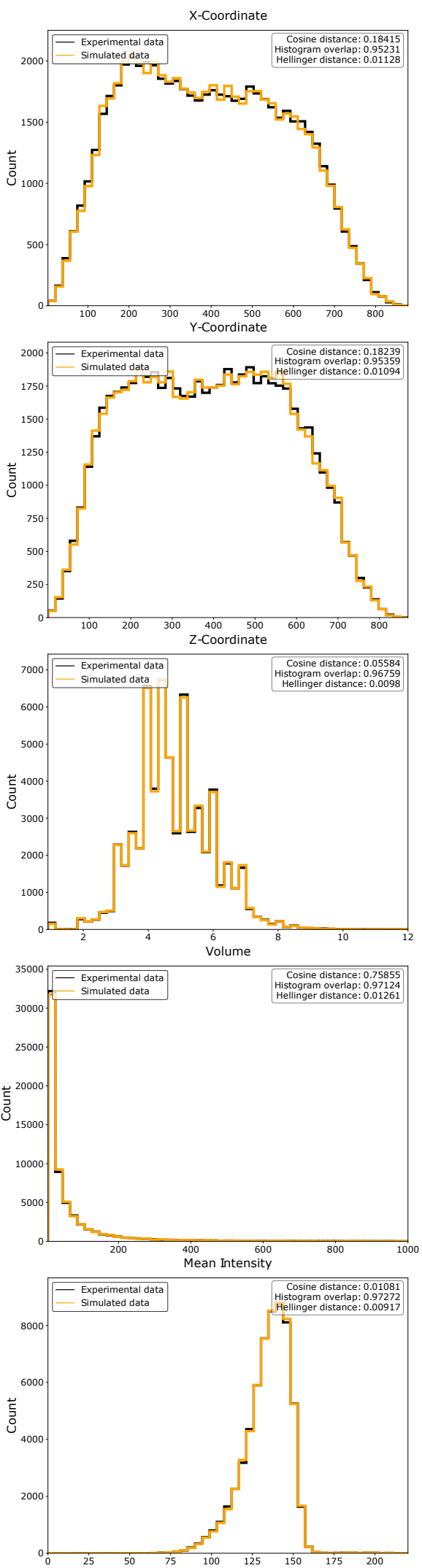

Control Circle BMPRIb simulated versus experimental data Inverse Transform Sampling check spread $n=0.0$
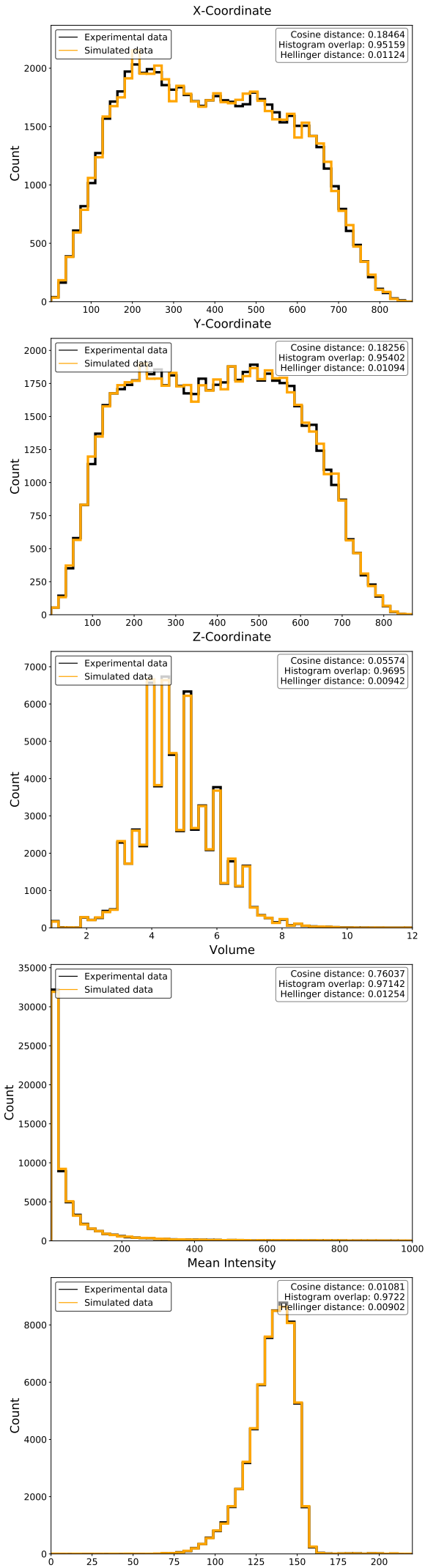

Figure A1. Histograms and similarity metrics of $x_{-}^{-}, y^{-}$, and $z$-coordinates, as well as the mean intensity and volume of Control Circle BMPRIb data with $\mathrm{n}=0$. 
Random division of experimental data into 2 groups for measure testing
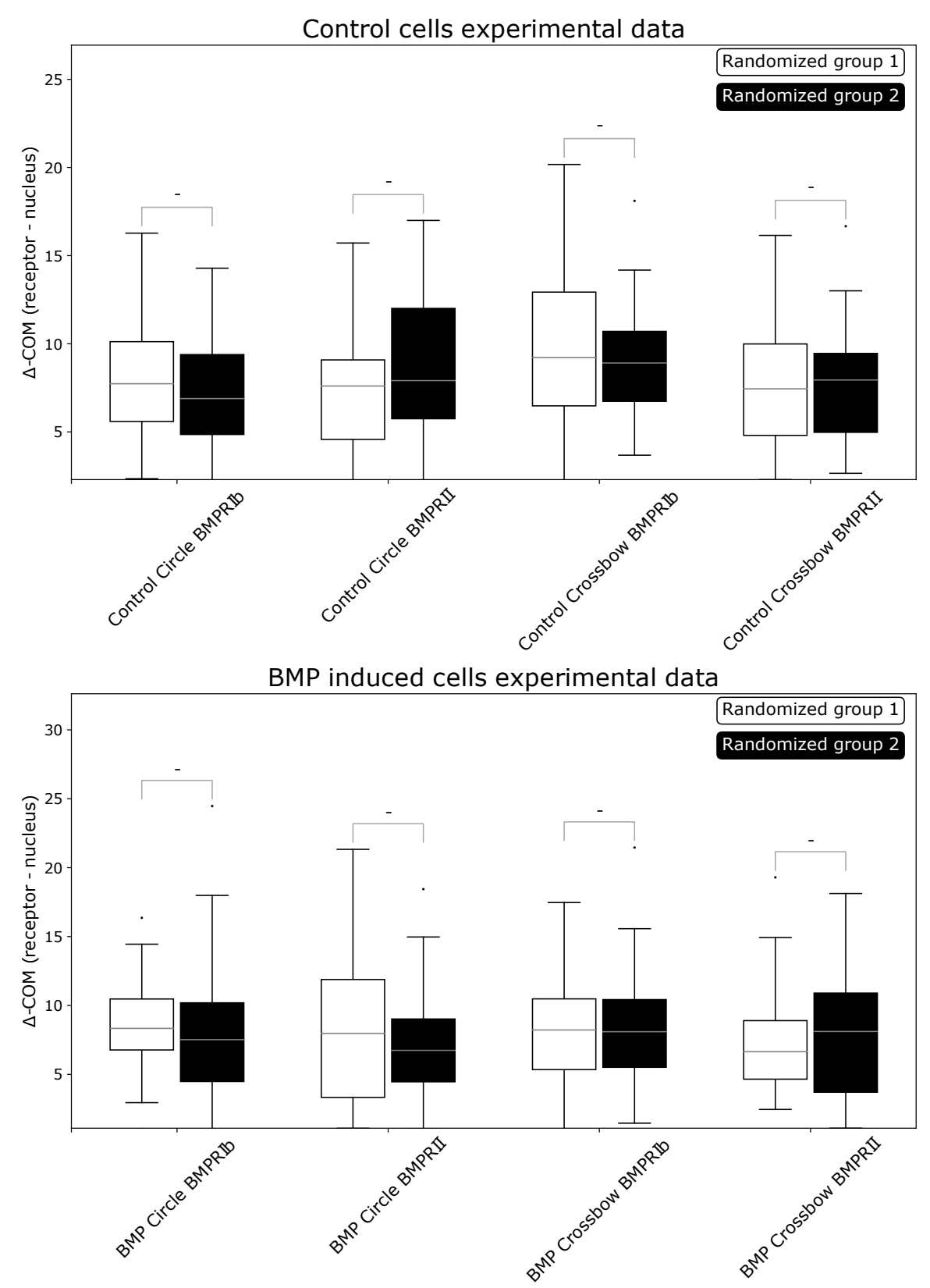

Figure A2. Comparison of experimental data randomly split into two groups for the reference metric. Then ComRed was used to assess the differences in the distributions. No significant differences were found. 
Random division of experimental data into 2 groups for measure testing
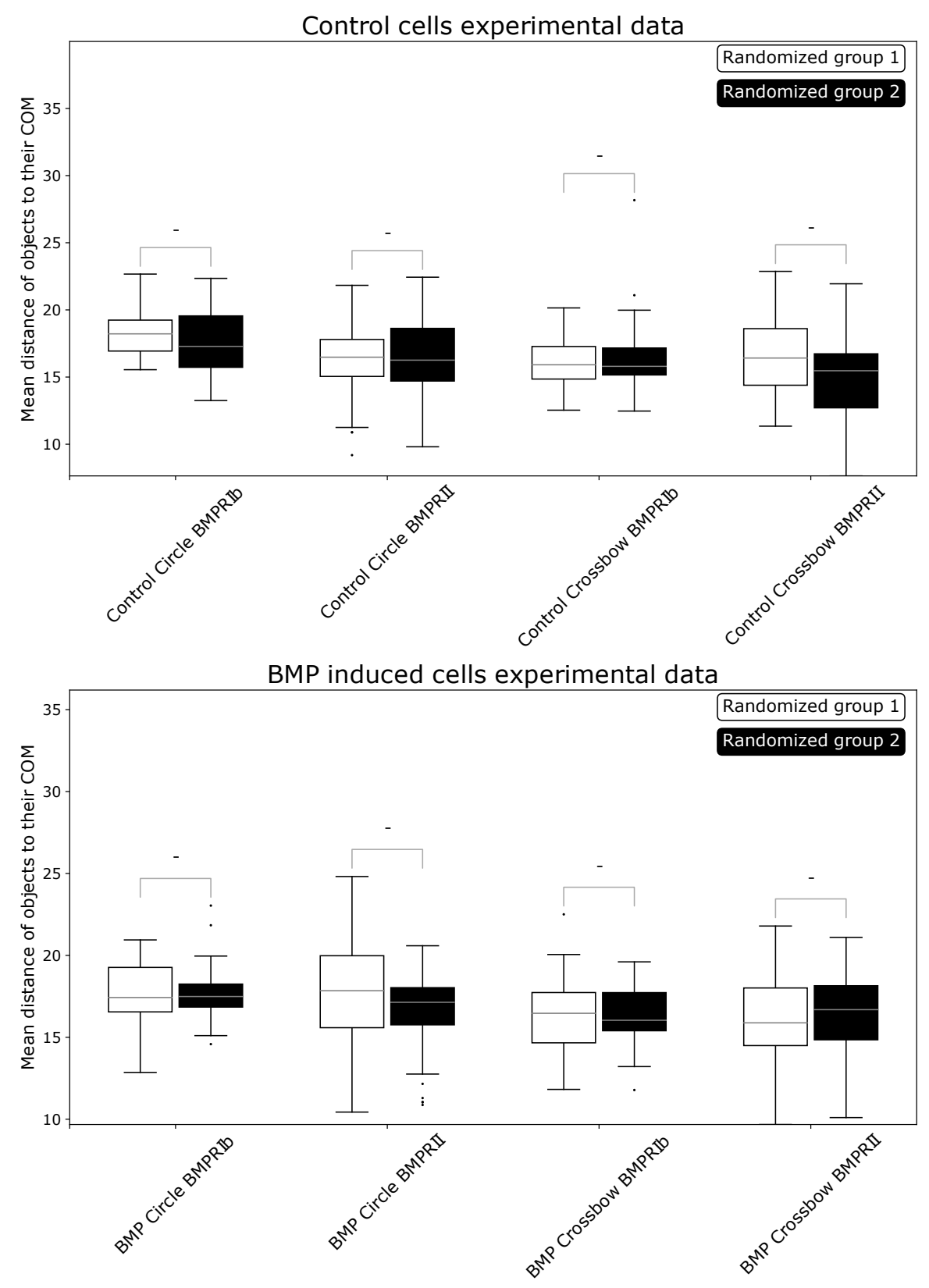

Figure A3. Comparison of experimental data randomly split into two groups for the shift metric. Then ComRed was used to assess the differences in the distributions. No significant differences were found. 

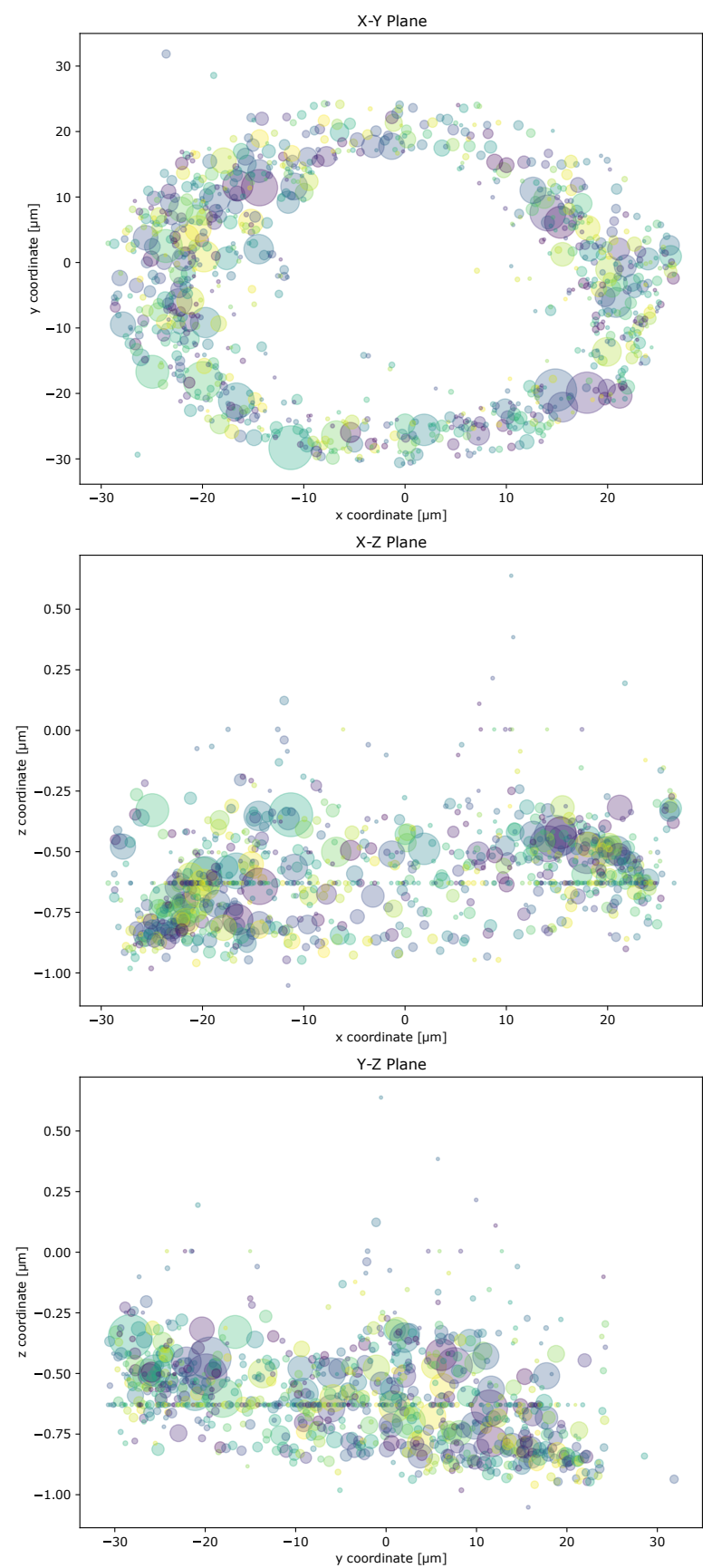

Figure A4. Visualization of the BMPRIb of an example control cell grown on a circular micropattern. Three projections of the receptor accumulation coordinates and virtual masses in the $x-y, x-z$, and $y-z$ planes. Virtual masses are indicated by the size of the dots. Different dot colors were chosen to differ between overlapping dots. These plots can be automatically generated by ComRed for checkup of found accumulations and their respective weight in the final center of mass. 
BMPs in regulating adipogenic and osteogenic differentiation of mesenchymal progenitor cells. Stem cells and development 2009, 18,545-559. doi:10.1089/scd.2008.0130.

2. Cunningham, N.S.; Paralkar, V.; Reddi, A.H. Osteogenin and recombinant bone morphogenetic protein 2B are chemotactic for human monocytes and stimulate transforming growth factor 81 mRNA expression. Proceedings of the National Academy of Sciences of the United States of America 1992, 89, 11740-11744.

3. Gamell, C.; Osses, N.; Bartrons, R.; Rückle, T.; Camps, M.; Rosa, J.L.; Ventura, F. BMP2 induction of actin cytoskeleton reorganization and cell migration requires PI3-kinase and Cdc42 activity. Journal of cell science 2008, pp. 3960-3970. doi:10.1242/jcs.031286.

4. Nohe, A.; Hassel, S.; Ehrlich, M.; Neubauer, F.; Sebald, W.; Henis, Y.I.; Knaus, P. The mode of bone morphogenetic protein (BMP) receptor oligomerization determines different BMP-2 signaling pathways. Journal of Biological Chemistry 2002, 277, 5330-5338. doi:10.1074/jbc.M102750200.

5. Hartung, A.; Bitton-Worms, K.; Rechtman, M.M.; Wenzel, V.; Boergermann, J.H.; Hassel, S.; Henis, Y.I.; Knaus, P. Different routes of bone morphogenic protein (BMP) receptor endocytosis influence BMP signaling. Molecular and cellular biology 2006, pp. 7791-7805. doi:10.1128/MCB.00022-06.

6. Pohl, T.L.M.; Boergermann, J.H.; Schwaerzer, G.K.; Knaus, P.; Cavalcanti-Adam, E.A. Surface immobilization of bone morphogenetic protein 2 via a self-assembled monolayer formation induces cell differentiation. Acta biomaterialia 2012, 8, 772-780. doi:10.1016/j.actbio.2011.10.019.

7. Migliorini, E.; Valat, A.; Picart, C.; Cavalcanti-Adam, E.A. Tuning cellular responses to BMP-2 with material surfaces. Cytokine $\mathcal{E}$ growth factor reviews 2016, 27, 43-54. doi:10.1016/j.cytogfr.2015.11.008.

8. Bragdon, B.; Thinakaran, S.; Bonor, J.; Underhill, T.M.; Petersen, N.O.; Nohe, A. FRET reveals novel protein-receptor interaction of bone morphogenetic proteins receptors and adaptor protein 2 at the cell surface. Biophysical journal 2009, 97, 1428-1435. doi:10.1016/j.bpj.2009.05.061.

9. Sieber, C.; Kopf, J.; Hiepen, C.; Knaus, P. Recent advances in BMP receptor signaling. Cytokine E growth factor reviews 2009, 20, 343-355. doi:10.1016/j.cytogfr.2009.10.007.

10. Paralkar, V.M.; Hammonds, R.G.; Reddi, A.H. Identification and characterization of cellular binding proteins (receptors) for recombinant human bone morphogenetic protein 2B, an initiator of bone differentiation cascade. Proceedings of the National Academy of Sciences of the United States of America 1991, 88, 3397-3401. doi:10.1073/pnas.88.8.3397.

11. Hardwick, J.C.H.; van den Brink, G.R.; Bleuming, S.A.; Ballester, I.; van den Brande, J.M.H.; Keller, J.J.; Offerhaus, G.J.A.; van Deventer, S.J.H.; Peppelenbosch, M.P. Bone morphogenetic protein 2 is expressed by, and acts upon, mature epithelial cells in the colon. Gastroenterology 2004, 126, 111-121. doi:10.1053/j.gastro.2003.10.067.

12. Benn, A.; Hiepen, C.; Osterland, M.; Schütte, C.; Zwijsen.; Knaus, P. Role of bone morphogenetic proteins in sprouting angiogenesis: differential BMP receptor-dependent signaling pathways balance stalk vs. tip cell competence. FASEB journal : official publication of the Federation of American Societies for Experimental Biology 2017, 31, 4720-4733. doi:10.1096/fj.201700193RR.

13. Zhou, J.; Lee, P.L.; Lee, C.I.; Wei, S.Y.; Lim, S.H.; Lin, T.E.; Chien, S.; Chiu, J.J. BMP receptor-integrin interaction mediates responses of vascular endothelial Smad1/5 and proliferation to disturbed flow. Journal of thrombosis and haemostasis : JTH 2013, 11, 741-755. doi:10.1111/jth.12159.

14. Benn, A.; Bredow, C.; Casanova, I.; Vukičević, S.; Knaus, P. VE-cadherin facilitates BMP-induced endothelial cell permeability and signaling. Journal of cell science 2016, 129, 206-218. doi:10.1242/jcs.179960.

15. Zuo, W.H.; Zeng, P.; Chen, X.; Lu, Y.J.; Li.; Wu, J.B. Promotive effects of bone morphogenetic protein 2 on angiogenesis in hepatocarcinoma via multiple signal pathways. Scientific reports 2016, p. 37499. doi:10.1038/srep37499.

16. Finkenzeller, G.; Hager, S.; Stark, G.B. Effects of bone morphogenetic protein 2 on human umbilical vein endothelial cells. Microvascular research 2012, 84, 81-85. doi:10.1016/j.mvr.2012.03.010.

17. Fernandez, S.M.; Berlin, R.D. Cell surface distribution of lectin receptors determined by resonance energy transfer. Nature 1976, 264, 411-415.

18. Algar, W.R.; Hildebrandt, N.; Vogel, S.S.; Medintz, I.L. FRET as a biomolecular research tool - understanding its potential while avoiding pitfalls. Nature methods 2019, 16, 815-829. doi:10.1038/s41592-019-0530-8.

19. Li, Q.; Lau, A.; Morris, T.J.; Guo, L.; Fordyce, C.B.; Stanley, E.F. A syntaxin 1, Galpha(o), and N-type calcium channel complex at a presynaptic nerve terminal: analysis by quantitative immunocolocalization. 
The Journal of neuroscience : the official journal of the Society for Neuroscience 2004, 24, 4070-4081. doi:10.1523/JNEUROSCI.0346-04.2004.

20. Bolte, S.; Cordelières, F.P. A guided tour into subcellular colocalization analysis in light microscopy. Journal of microscopy 2006, 224, 213-232. doi:10.1111/j.1365-2818.2006.01706.x.

21. Medda, R.; Giske, A.; Cavalcanti-Adam, E.A. Challenges in imaging cell surface receptor clusters. Optics and Lasers in Engineering 2016, 76, 3-8. doi:10.1016/j.optlaseng.2015.03.020.

22. Bacia, K.; Kim, S.A.; Schwille, P. Fluorescence cross-correlation spectroscopy in living cells. Nature methods 2006, 3, 83-89. doi:10.1038/nmeth822.

23. Lippincott-Schwartz, J.; Snapp, E.L.; Phair, R.D. The Development and Enhancement of FRAP as a Key Tool for Investigating Protein Dynamics. Biophysical journal 2018, 115, 1146-1155. doi:10.1016/j.bpj.2018.08.007.

24. Nohe, A.; Keating, E.; Underhill, T.M.; Knaus, P.; Petersen, N.O. Effect of the distribution and clustering of the type I A BMP receptor (ALK3) with the type II BMP receptor on the activation of signalling pathways. Journal of cell science 2003, pp. 3277-3284. doi:10.1242/jcs.00519.

25. Théry, M. Micropatterning as a tool to decipher cell morphogenesis and functions. Journal of cell science 2010, 123, 4201-4213. doi:10.1242/jcs.075150.

26. Sommer, C.; Straehle, C.; Köthe, U.; Hamprecht, F.A. ilastik: Interactive Learning and Segmentation Toolkit. Eighth IEEE International Symposium on Biomedical Imaging (ISBI). Proceedings 2011, pp. 230-233.

27. Berg, S.; Kutra, D.; Kroeger, T.; Straehle, C.N.; Kausler, B.X.; Haubold, C.; Schiegg, M.; Ales, J.; Beier, T.; Rudy, M.; Eren, K.; Cervantes, J.I.; Xu, B.; Beuttenmueller, F.; Wolny, A.; Zhang, C.; Koethe, U.; Hamprecht, F.A.; Kreshuk, A. ilastik: interactive machine learning for (bio)image analysis. Nature methods 2019, 16, 1226-1232. doi:10.1038/s41592-019-0582-9.

28. Théry, M.; Racine, V.; Piel, M.; Pépin, A.; Dimitrov, A.; Chen, Y.; Sibarita, J.B.; Bornens, M. Anisotropy of cell adhesive microenvironment governs cell internal organization and orientation of polarity. Proceedings of the National Academy of Sciences of the United States of America 2006, 103, 19771-19776. doi:10.1073/pnas.0609267103.

29. Jortikka, L.; Laitinen, M.; Lindholm, T.; Marttinen, A. Internalization and Intracellular Processing of Bone Morphogenetic Protein (BMP) in Rat Skeletal Muscle Myoblasts (L6). Cellular Signalling 1997, 9, 47-51. doi:10.1016/S0898-6568(96)00094-0.

30. O'Sullivan, M.J.; Lindsay, A.J. The Endosomal Recycling Pathway-At the Crossroads of the Cell. International journal of molecular sciences 2020, 21. doi:10.3390/ijms21176074.

31. Mitchell, H.; Choudhury, A.; Pagano, R.E.; Leof, E.B. Ligand-dependent and -independent transforming growth factor-beta receptor recycling regulated by clathrin-mediated endocytosis and Rab11. Molecular Biology of the Cell 2004, 15, 4166-4178. doi:10.1091/mbc.e04-03-0245.

32. Lin, H.Y.; Wang, X.F.; Ng-Eaton, E.; Weinberg, R.A.; Lodish, H.F. Expression and cloning of the TGF- $\beta$ Type II Receptor, a Functional Transmembrane Serine/Threonine Kinase. Cell 1992, 68, 775-785.

33. Franzén, P.; ten Dijke, P.; Ichijo, H.; Yamashita, H.; Schulz, P.; Heldin, C.H.; Miyazono, K. Cloning of a TGF $\beta$ Type I Receptor That Forms a Heteromeric Complex with the TGF $\beta$ Type II Receptor. Cell 1993, 75, 681-692.

34. Di Guglielmo, G.M.; Le Roy, C.; Goodfellow, A.F.; Wrana, J.L. Distinct endocytic pathways regulate TGF-beta receptor signalling and turnover. Nature cell biology 2003, 5, 410-421. doi:10.1038/ncb975.

35. Gleason, R.J.; Akintobi, A.M.; Grant, B.D.; Padgett, R.W. BMP signaling requires retromer-dependent recycling of the type I receptor. Proceedings of the National Academy of Sciences of the United States of America 2014, 111, 2578-2583. doi:10.1073/pnas.1319947111.

36. Schindelin, J.; Arganda-Carreras, I.; Frise, E.; Kaynig, V.; Longair, M.; Pietzsch, T.; Preibisch, S.; Rueden, C.; Saalfeld, S.; Schmid, B.; Tinevez, J.Y.; White, D.J.; Hartenstein, V.; Eliceiri, K.; Tomancak, P.; Cardona, A. Fiji: an open-source platform for biological-image analysis. Nature methods 2012, 9, 676-682. doi:10.1038/nmeth.2019. 